\title{
EFICIÊNCIA DE ISCA FORMICIDA APLICADA SOBRE O MONTE DE TERRA SOLTA DE NINHOS DE Atta sexdens rubropilosa (HYMENOPTERA: FORMICIDAE) ${ }^{1}$
}

\author{
Ronald Zanetti², José Cola Zanuncio ${ }^{3}$, Alan Souza-Silva ${ }^{2}$ e Luiz Gonzaga de Abreu ${ }^{4}$
}

\begin{abstract}
RESUMO - Avaliou-se a eficiência de uma isca granulada à base de sulfluramida $(0,3 \%)$ aplicada em olheiros situados sobre o monte de terra solta, em comparação com o sistema convencional de aplicação nos olheiros fora desses montes, no combate a Atta sexdens rubropilosa Forel, 1908 (Hymenoptera: Formicidae). Os tratamentos consistiram da aplicação de uma isca granulada à base de sulfluramida $(0,3 \%)$, a $8 \mathrm{~g} / \mathrm{m}^{2}$ do formigueiro aparente, em olheiros situados sobre ou fora do monte de terra solta. Foram utilizados 192 formigueiros de A. sexdens rubropilosa, distribuídos nesses dois tratamentos. Foram avaliadas a porcentagem de carregamento e de devolução dessa isca, aos dois dias após sua aplicação, e a eficiência do combate, aos 150 dias após, por sondagem (uma a cada metro quadrado de fomigueiro). A porcentagem de carregamento da isca granulada foi maior quando aplicada fora do monte de terra solta $(100,00 \%)$ do que sobre ele $(89,58 \%)$, porém a eficiência de controle foi semelhante, com $89,58 \%$ para o primeiro e $90,27 \%$ para o segundo tratamento, respectivamente. Por isto, em razão do menor custo e facilidade, recomenda-se aplicar esta isca sobre os montes de terra solta de formigueiros de A. sexdens rubropilosa.
\end{abstract}

Palavras-chave: Isca granulada, métodos de aplicação, formigas-cortadeiras e monte de terra solta.

\section{EFFICIENCY OF A GRANULATED BAIT APPLIED OVER THE NESTS OF Atta sexdens rubropilosa (HYMENOPTERA: FORMICIDAE)}

\begin{abstract}
The efficiency of a granulated bait formulated with sulfluramid (0.3\%) for the control of Atta sexdens rubropilosa Forel, 1908 (Hymenoptera: Formicidae) was evaluated. Treatments consisted of applying this bait at a dose of $8 \mathrm{~g} / \mathrm{m}^{2}$ from the ant nest. This bait was applied near entrances over ant nests (treatment T1) or near those outside these nests (treatment T2). A total of 192 ant nests of A. sexdens rubropilosa were used in the two treatments. Percentage of transport and devolution of the bait by A. sexdens rubropilosa was evaluated two days after its application and control efficiency, 150 days after application. Percentage of bait transport was higher when applied outside the ant nest (100.00\%) than over it (89.5\%). However, control efficiency was similar between treatments. For this reason and due to lower costs, it is recommended to apply this bait near entrances over ant colonies to control A. sexdens rubropilosa.
\end{abstract}

Key words: Granulated bait, application methods, leaf cutting ants and ant nest.

1 Recebido para publicação em 21.2.2002.

Aceito para publicação em 12.5.2003.

2 Departamento de Entomologia da Universidade Federal de Lavras - UFLA, Caixa Postal 37, 37200-000 Lavras-MG, <zanetti@ufla.br>; <alandesouza@hotmail.com>. ${ }^{3}$ Departamento de Biologia Animal da Universidade Federal de Viçosa - UFV, 36571-000 Viçosa-MG, <zanuncio@ mail.ufv.br>. ${ }^{4}$ Acesita Energética, Timóteo-MG. 


\section{INTRODUCÃO}

As formigas-cortadeiras dos gêneros Atta e Acromyrmex apresentam sistema de vida com organização social semelhante, vivem em ninhos subterrâneos e são as principais pragas florestais na América (Della Lucia, 1993). Esses ninhos são formados por uma ou mais câmaras, onde se encontram o jardim de fungo, a rainha e a prole dessas formigas (Zanetti et al., 2002). As câmaras ou panelas são interligadas por canais ou galerias e a saídas para a superfície do solo, que se denominam olheiros. Estes olheiros podem localizar-se sobre os montes de terra solta formados pelo acúmulo de solo extraído das câmaras, mas, também, como saídas fora deles, os quais são chamados olheiros de abastecimento, de carregamento ou de ventilação (Della Lucia, 1992).

Os processos de combate a formigas-cortadeiras incluem diferentes métodos, que variaram ao longo do tempo e podem ser classificados de acordo com a formulação do formicida aplicado (Nogueira et al., 1981). Em áreas florestais cultivadas, as iscas granuladas são preferidas para o controle de formigas-cortadeiras, por apresentarem menor custo de combate e mais facilidade de aplicação que outros métodos de controle. Essas iscas podem ser aplicadas diretamente nos formigueiros (Laranjeiro, 1988; Zanuncio et al., 1992), ou em saquinhos plásticos distribuídos no reflorestamento em dose única ou por metro quadrado de formigueiro (Laranjeiro \& Zanuncio, 1995). A sulfluramida é o princípio ativo mais usado em iscas formicidas e apresenta eficiência elevada contra formigas-cortadeiras (Cameron, 1990; Cruz et al., 1996, 2000; Zanuncio et al., 2000).

As orientações técnicas de combate recomendam a aplicação de iscas granuladas nos olheiros de abastecimento situados fora dos montes de terra solta dos ninhos de formigas-cortadeiras. No entanto, em florestas implantadas, com sub-bosque mais denso, a localização desses olheiros é mais difícil, o que reduz o rendimento e aumenta o custo dessa operação (Zanuncio et al., 1992). Além disto, em áreas com grande densidade de sauveiros próximos uns dos outros é mais difícil definir a qual formigueiro pertence o olheiro selecionado para aplicação da isca. Neste caso, a isca poderá ser aplicada em olheiros de outros formigueiros, ocasionando subdosagem no ninho-alvo e diminuição da eficiência do combate. Uma solução para esse problema seria a aplicação da isca em olheiros situados sobre os montes de terra solta, que são mais facilmente localizados que aqueles situados fora deles e não despertam dúvidas sobre o formigueiro a que pertencem (Zanuncio et al., 1992).

Considerando a alta eficiência de uma isca granulada à base de sulfluramida $(0,3 \%)$ para diversas espécies de formigas-cortadeiras (Cameron, 1990; Cruz et al., 1996, 2000; Zanuncio et al., 2000), objetivou-se avaliar a eficiência dessa isca aplicada em olheiros situados sobre montes de terra solta, em comparação com sua aplicação em olheiros de abastecimento fora desses montes, no controle da formiga cortadeira Atta sexdens rubropilosa Forel, 1908 (Hymenoptera: Formicidae) em plantio de eucalipto.

\section{MATERIAL E MÉTODOS}

Foram selecionados 192 formigueiros de $A$. sexdens rubropilosa, com área de terra solta aparente variando de 8 a $132 \mathrm{~m}^{2}$ em quatro talhões de eucalipto, com área total de 200 ha de Eucalyptus urophylla S.T. Blake com aproximadamente 4 anos de idade, da Acesita Energética, no município de Timóteo, Minas Gerais. Tais formigueiros foram medidos, multiplicando-se o maior comprimento pela maior largura da área de terra solta aparente dos ninhos, e distribuídos em igual número e aleatoriamente em dois tratamentos, seguindo o delineamento inteiramente casualizado. O primeiro consistiu da aplicação da isca granulada à base de sulfluramida $(0,3 \%)$, na dosagem de $8 \mathrm{~g}$ por metro quadrado aparente de formigueiro (Mariconi, 1970), em olheiros situados sobre o monte de terra solta; o segundo consistiu na aplicação dessa isca, na mesma dosagem, porém distribuída em olheiros fora do monte de terra solta de formigueiros de $A$. sexdens rubropilosa. Foram avaliados o carregamento e a devolução dessa isca aos dois dias após sua aplicação e a eficiência de controle dessa formiga-cortadeira 150 dias após, através da abertura dos formigueiros com enxadão e sondagem com sonda JP (Cruz et al., 1996), para verificar a sua mortalidade.

Os dados sobre a eficiência de controle de $A$. sexdens rubropilosa foram submetidos à análise não-paramétrica de Kruskall-Wallis, indicada por Sokal \& Rohf (1969) para dados em escala nominal de valores (ativos ou inativos). Nesta análise, as médias das ordens de cada tratamento foram confrontadas duas a duas, em nível de 5\% de probabilidade, para se determinar diferenças significativas entre tratamentos (Gomes, 1985). Os dados sobre o carregamento e a devolução da isca foram analisados em porcentagem. 


\section{RESULTADOS E DISCUSSÃO}

A porcentagem de carregamento da isca granulada à base de sulfluramida foi maior quando aplicada fora do monte de terra solta $(100,00 \%)$ do que quando aplicada sobre ele $(89,58 \%)$ (Kruskal-Wallis, $\mathrm{p}<0,05)$. Este fato pode ser explicado pela maior atividade de forrageamento que ocorre, normalmente, em olheiros de abastecimento do que naqueles de limpeza. Apesar disto, a eficiência de controle foi semelhante nas duas metodologias de aplicação da isca, com 90,27\% para os sauveiros que receberam essa isca sobre o monte de terra solta e $89,58 \%$ para os que a receberam fora do monte (Quadro 1), o que mostra que a aplicação da isca granulada sobre o monte de terra solta é tão eficiente no controle de $A$. sexdens rubropilosa quanto à sua aplicação fora dele. Portanto, recomenda-se a utilização do primeiro método, pelo fato de seu custo de aplicação ser menor, pois os olheiros que receberão a isca são mais visíveis. Desta forma, o operador economiza tempo na localização dos olheiros de limpeza que receberão isca, pois os de abastecimento são mais difíceis de ser localizados, especialmente em área de sub-bosque mais denso ou onde haja muitos detritos. Além disto, diminui-se a possibilidade de aplicação da isca em olheiros de outros formigueiros próximos, evitando-se a subdosagem do ninhoalvo e a diminuição da eficiência do combate.

Embora a eficiência da isca granulada com sulfluramida tenha sido semelhante nas duas metodologias, sua

Quadro 1 - Eficiência da isca granulada à base de sulfluramida aplicada sobre ou fora do monte de terra solta no controle de formigueiros de Atta sexdens rubropilosa (Hymenoptera: Formicidae). Timóteo, Minas Gerais, Brasil

Table 1 - Efficiency of the granulated bait formulated with sulfluramid applied over and outside anthills, against Atta sexdens rubropilosa (Hymenoptera: Formicidae). Timóteo, Minas Gerais, Brazil

\begin{tabular}{|c|c|c|c|c|}
\hline Tratamento & $\begin{array}{c}\text { Área } \\
\text { Média dos } \\
\text { Formi- } \\
\text { gueiros } \\
\left(\mathrm{m}^{2}\right)\end{array}$ & $\begin{array}{c}\mathrm{N} \text { o de } \\
\text { Olheiros/ } \\
\text { Formi- } \\
\text { gueiro }\end{array}$ & $\begin{array}{c}\text { Porcenta- } \\
\text { gem de } \\
\text { Carrega- } \\
\text { mento }\end{array}$ & $\begin{array}{c}\text { Eficiência } \\
\text { do } \\
\text { Controle } \\
(\%)^{*}\end{array}$ \\
\hline Sobre o Monte & 21,52 & 5,19 & 89,58 & 90,27 \\
Fora do Monte & 36,45 & 6,66 & 100,00 & 89,58 \\
\hline
\end{tabular}

* não há diferença entre as médias dos tratamentos (Kruskal Wallis, $\mathrm{p}>0,05)$. aplicação sobre o monte de terra solta diminui o tempo de controle, por não haver necessidade de se procurar os olheiros ao redor dos ninhos. Tal fato permite uma distribuição mais uniforme da isca que aquela da forma convencional, de aplicação fora dos montes, pois cada formigueiro receberia, proporcionalmente, quantidades adequadas de isca em relação à sua área de terra solta. Deve-se ressaltar, ainda, que cada monte de terra solta recebe isca granulada, evitando-se que montes de tamanho reduzido e que poderiam ser formigueiros iniciais não recebessem esse tipo de controle.

A alta eficiência de controle observada concorda com relatos para diversas espécies de formigas-cortadeiras como Atta sexdens sexdens (Cruz et al., 1996), A. sexdens rubropilosa (Zanuncio et al., 2000), Atta laevigata (Alves et al., 1997), Atta cephalotes (Zanuncio et al., 1996) e Acromyrmex octospinosus (Cruz et al., 2000). No entanto, a eficiência dessa isca granulada aplicada sobre o monte de terra solta de formigueiros de A. sexdens rubropilosa, em relação à sua aplicação convencional, em olheiros fora desses montes, não havia sido ainda estudada.

\section{CONCLUSÃO}

A aplicação da isca granulada à base de sulfluramida sobre montes de terra solta é tão eficiente no combate à formiga-cortadeira $A$. sexdens rubropilosa quanto a sua aplicação em olheiros fora deles. Por isto, recomenda-se o uso dessa metodologia, com o cuidado de que essa isca granulada seja aplicada no lado contrário àquele em que as formigas estejam depositando a terra escavada, para evitar que ela seja coberta por terra.

\section{REFERÊNCIAS BIBLIOGRÁFICAS}

ALVES, J. B. et al. Paralisação de forrageamento e controle de Atta laevigata (F. Smith) (Hymenoptera: Formicidae) com Mirex-S (sulfluramida) em duas metodologias de medição de formigueiros. Revista Árvore, v. 21, n. 1, p. 141-146, 1997.

CAMERON, R. S. Potential baits for control of the Texas leaf-cutting ant, Atta texana (Hymenoptera: Formicidae). In: VANDER MEER, R. K.; JAFF, E. K.; CEDENO, A. (Eds.) Applied Myrmecology: a world perspective. 1990. p. 628-37.

CRUZ, A. P. et al. Eficiência de iscas à base de sulfluramida e de clorpirifós no controle de Atta sexdens sexdens (Hymenoptera: Formicidae), no trópico úmido. Acta Amazonica, v. 26, n. 3, p. 145-150, 1996.

R. Árvore, Viçosa-MG, v.27, n.3, p.407-410, 2003 
CRUZ, A. P. et al. Eficiencia de cebos a base de sulfluramida o de clorpirifós en el control de Acromyrmex octospinosus (Hymenoptera: Formicidae) en el trópico húmedo. Revista Colombiana de Entomología, v. 26, n. 1-2, p. 67-69, 2000.

DELLA LUCIA, T. M. C. Bioecologia e controle de formigas cortadeiras. In: REUNIÃO SOBRE PRAGAS SUBTERRÂNEAS DOS PAÍSES DO CONE SUL, 2., 1992, Sete Lagoas. Anais... Sete Lagoas: 1992. p. 35-45.

DELLA LUCIA, T. M. C. (Ed.). As formigas cortadeiras. Viçosa: Folha de Viçosa, 1993. 262 p.

GOMES, F. P. Curso de estatística experimental. Piracicaba: Nobel, 1985. 467 p.

LARANJEIRO, A. J. Controle de formigas em reflorestamentos: propagação, operação e monitoramento. In: CURSO DE ATUALIZAÇÃO NO CONTROLE DE FORMIGAS CORTADEIRAS, 2., 1988. Piracicaba.

Curso... Piracicaba: 1988. 24 p.

LARANJEIRO, A. J.; ZANUNCIO, J. C. Avaliação da isca à base de sulfluramida no controle de Atta sexdens rubropilosa pelo processo de dosagem única de aplicação. IPEF, v. 48/49, p. 144-152, 1995.
MARICONI, F. A. M. As saúvas. São Paulo: Agronômica Ceres, 1970. 167 p.

NOGUEIRA, S. B.; CALIL, A. C. P.; BARRIGOSSI, J. A. F. Formicidas termonebuláveis no combate às espécies de saúvas, Atta sexdens rubropilosa, A. laevigata e $A$. bisphaerica (Formicidae: Hymenoptera). Revista Seiva, v. 41, n. 89, p. 23-27, 1981.

SOKAL, R. R.; ROHLF, F. J. Biometry: the principles and practice of statistics in biological research. San Francisco: W.H. Freeman and Company, 1969. 776 p.

ZANETTI, R. et al. Manejo integrado de formigas cortadeiras. Lavras: UFLA, 2002. 16 p.

ZANUNCIO, J. C. et al. Eficiência da isca granulada Mirex-S, à base de sulfluramida no controle da formiga cortadeira Atta laevigata (F. Smith, 1858) (Hymenoptera: Formicidae). Revista Árvore, v. 16, n. 3, p. 357-361, 1992.

ZANUNCIO, J. C. et al. Eficiência da isca Mirex-S (sulfluramida 0,3\%), no controle de Atta cephalotes (Hymenoptera: Formicidae) em três dosagens. Acta Amazônica, v. 26, n. 1/2, p. 115-120. 1996.

ZANUNCIO, J. C. et al. Utilización del cebo Mirex-S (Sulfluramida $0.3 \%$ ) para el control de Atta sexdens rubropilosa (Hymenoptera: Formicidae) en área estratificada de hormigueros. Revista Colombiana de Entomologia, v. 26, n. 3-4, p. 157-160, 2000. 